\title{
Biological Applications of Micro-Raman Spectroscopy
}

\author{
W. Kiefer*, M. Schmitt*, R. Gessner*, R. Rösch**, J. Popp** \\ * Institut für Physikalische Chemie, Universität Würzburg, Am Hubland, D-97074 Würzburg, \\ Germany \\ ** Institut für Physikalische Chemie, Universität Jena, Helmholtzweg 4, D-07743 Jena, Germany
}

During the last couple of years in-vivo Raman spectroscopical investigations of biological tissues and cells became of significant importance in the fields of medical, pharmaceutical and biological diagnosis. In this context the chemical composition of cell material and its dependency on exogenous factors are of utmost importance. Therefore it must be the task of in-vivo methods to provide this information about the cell ingredients of living cells without destroying them. MicroRaman spectroscopy appears to be the most suitable technique for such scientific investigations. For example, water which shows sharp absorptions in the infrared is only a weak Raman scatterer. Thus biological tissues containing a high amount of water can be easily studied with Raman spectroscopy. Due to the use of laser light for the excitation of Raman scattering sub- $\mu \mathrm{m}$ spatial resolution can be realized by micro-Raman spectroscopy. This allows the investigation of very heterogeneous samples. The main focus of our work lies on the investigation of secondary metabolites such as alkaloids, lipis or terpenes, which agglomerate in the plant. Different components in tobacco plants (N. tabakum and N. rustica) were identified. Micro-Raman spectroscopy has also been used for the characterization of oil droplets in the xylem of several plants (Myrothamnus flabellifolia, poplar lignin and cellulose). Another aspect of our work was the characterization of essential oils of laminacea plants and citrus fruits directly at the point of origin, the glandular trichromes or lysigeic oil cavities. The composition of an essential oil as well as the concentration of its components changes due to environmental influences. To study the influence of the general cultivation conditions three types of mints were cultivated outdoors as well as under sterile conditions. Additionally, the composition of the essential oil shows annual variations. This is exemplarily shown for the bergamont mint. Another aim of these investigations is the realization of a chemotaxonomic classification of plants. Therefore, a hierarchical cluster analysis was performed with the micro-Raman spectra of the single plants.

All these results show, that Raman spectroscopy is well suited for identifying compounds of biological material but when working with biological samples, several problems may arise: (1) the very small substance quantities are often below the detection limit of Raman spectroscopy, (2) a lot of biological samples show fluorescence that is orders of magnitude more intense than the Raman signal, (3) biological samples are very sensitive and might be destroyed by the Raman excitation laser. One method to overcome these problems is the surface-enhanced Raman scattering (SERS) technique. However, the high amount of heavy metal (e.g., Ag, $\mathrm{Au}, \mathrm{Cu}$ ) required for the SERS technique often leads to the destruction of the highly sensitive biological samples. In order to reduce the metal concentration we used silver-coated glass fiber tips with diameters in the submicron size range as the SERS substrate. An additional major advantage of using these glass fiber tips is the possibility of exact positioning of the tip and therefore the exciting laser to the designated area of the biological sample to be investigated. Due to the small dimensions of the fiber and the fact that the main light intensity is emanating from the fiber tip, the spatial resolution of this method is very good. This was verified by an experiment with crystal violet solution as a model substance where a spatial resolution of 200--500 nm, depending on the tip that was used could be reached. An 
impressive example for the practicability of this method is the investigation of monolayers of the yeast Rhodotorula mucilaginosa (rubra) on a glass slide. The main problem with laser spectroscopy on microorganisms is their sensitivity towards photoinduced destruction. Figure 1(a) shows the attempt of an area scan with a conventional micro-Raman setup on a smear of the yeast $R$.

mucilaginosa on a glass slide. It can be seen that the area scanned by the laser is practically cleansed of all yeast cells and no spectrum could be obtained by this method. A micro-Raman spectrum was therefore recorded directly from the agar plate on which the yeast was grown. The multilayered cell colonies could withstand the laser long enough to provide spectrum B shown in Fig. 1(c). Figure 1(b) shows a glass fiber tip at a small yeast cell colony on a glass slide. With the fiber setup shown in Fig.1(b) it was possible to obtain spectrum C in Fig. 1(c) without destroying the yeast cells during the measurement. Therefore, it should be possible to measure microorganisms directly from contaminated surfaces without growing pure strains. Spectrum A in Fig. 1C shows a bulk spectrum of $\beta$-carotene in ethanol as a reference. The slight shift that is observable between the ethanolic $\beta$ carotene spectrum and the spectrum of $R$. mucilaginosa is due to the different environments in which the spectra are recorded. It is possible to obtain spectra from the microorganisms with fiber tips due to the considerably reduced laser power.
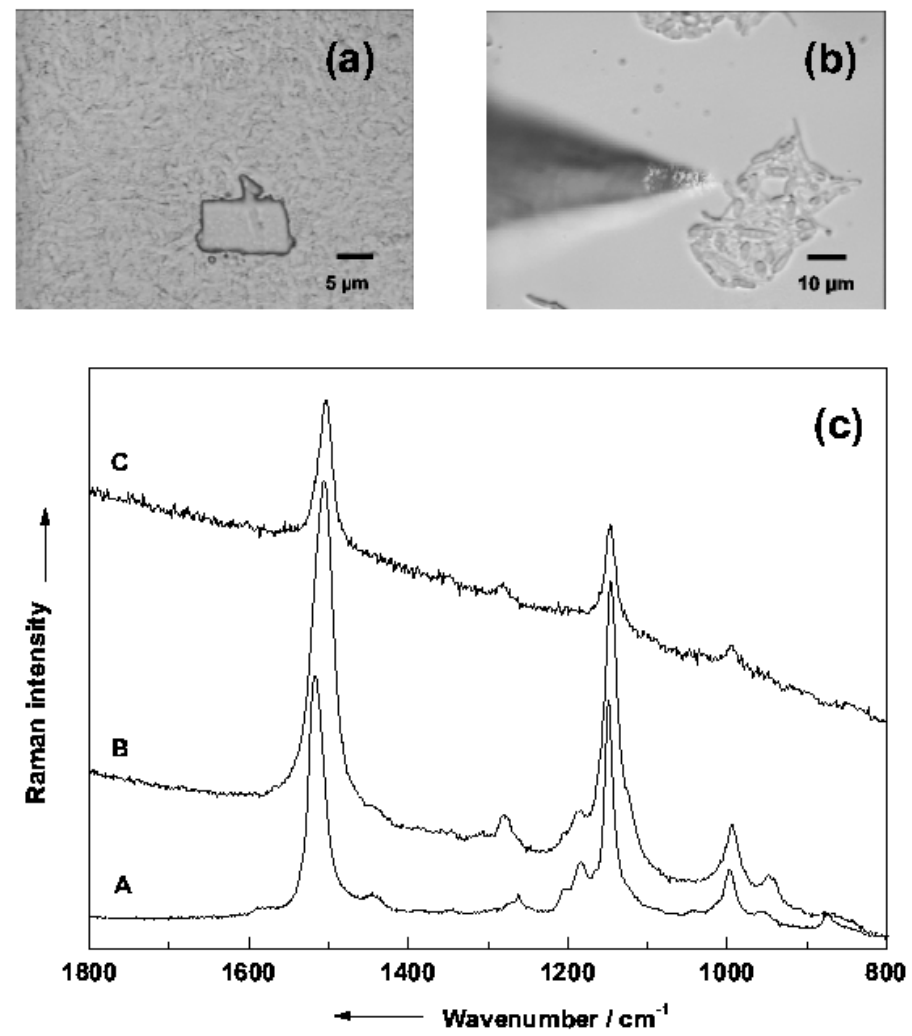

Fig. 1: A) Microphotograph of a smear of the yeast Rhodotorula mucilaginosa on a glass slide. The erased area was destroyed by the laser. B) Illuminated SERS fiber tip in close proximity of a small yeast colony, and $\mathrm{C}$ ) the Raman spectra taken from $\beta$-carotene and of the yeast $R$. mucilaginosa under differing experimental conditions: $\beta$-carotene in ethanol, $10 \mathrm{~mm}$ sample cell, micro-Raman setup, $60 \mathrm{~mW}$ laser power on the sample (a), R. mucilaginosa on agar, micro-Raman setup, $60 \mathrm{~mW}$ laser power on the sample (b), and a small yeast colony of $R$. mucilaginosa prepared on a glass slide, SERS fiber tip, $0.2 \mathrm{~mW}$ laser power on the sample (c). 